\title{
Selective Growth of PbSe on One or Both Tips of Colloidal Semiconductor Nanorods
}

\author{
Stefan Kudera, ${ }^{\dagger}$ Luigi Carbone, ${ }^{\ddagger}$ Maria Francesca Casula, ${ }^{\S}$ Roberto Cingolani,, \\ Andrea Falqui,\& Etienne Snoeck," Wolfgang J. Parak, ${ }^{*, \dagger}$ and Liberato Manna, ${ }^{*, \neq}$ \\ Center for Nanoscience, Ludwig Maximilians Universität München, Munich, Germany, \\ National Nanotechnology Laboratory of INFM, Via Arnesano Km 5, \\ 73100 Lecce, Italy, Dipartimento di Scienze Chimiche, Università di Cagliari, \\ 09042 Monserrato, Cagliari, Italy, and CEMES/CNRS, 29 Rue Jeanne Marvig, \\ 31055 Toulouse, France
}

Received November 23, 2004; Revised Manuscript Received January 13, 2005

\begin{abstract}
PbSe nanocrystals with rock-salt structure are grown on the tips of colloidal CdS and CdSe nanorods. The facets of wurtzite rods provide a substrate with various degrees of reactivity for the growth of PbSe. The presence of dangling Cd bonds may explain subtle differences between nonequivalent facets resulting in the selective nucleation of PbSe only on one of the two tips of each CdS rod. This approach has the potential to facilitate the fabrication of heterostructures with tailored optical and electronic properties.
\end{abstract}

Architectures based on inorganic materials, such as selfassembled quantum dots, ${ }^{1}$ nanowires, ${ }^{2}$ and nanowire superlattices, ${ }^{3,4}$ arise from the delicate balance, at the nanoscale, of parameters such as strain, surface and interfacial energy, and solubility. An increasing degree of complexity is being achieved in such structures, as they start incorporating multiple materials linked together in a programmed way. ${ }^{5}$ Likewise, research on colloidal nanocrystals has moved from the synthesis of simple structures, such as spherical nanoparticles, to more elaborate shapes such as rods, ${ }^{6,7}$ stars, ${ }^{8}$ disks, ${ }^{9}$ branched nanocrystals ${ }^{10-12}$ and recently to nanostructures based on inorganic sections interconnected without the need of organic linkers. ${ }^{13-15}$ Recent examples are inorganically coupled quantum rods and dots connected epitaxially at branched and linear junctions within single colloidal nanocrystals, ${ }^{13}$ metal-tipped semiconductor nanorods, ${ }^{14}$ and nanocrystal heterodimers based on magnetic and fluorescent nanoparticles. ${ }^{15}$

Nanocrystal heterostructures represent a convenient approach to the development of nanoscale building blocks, ${ }^{11}$ as they group inorganic sections with different functionalities in the same particle. Sections based on semiconductor nanorods, for instance, will emit linearly polarized light, ${ }^{16,17}$ will

* Corresponding authors: Wolfgang.Parak@physik.uni-muenchen.de; tel +49 (0)89 21801438; fax +49 (0)89 21802050. liberato.manna@unile.it; tel. +390832298 207; fax +390832 298238 .

$\dagger$ Ludwig Maximilians Universität München.

$\doteqdot$ National Nanotechnology Laboratory of INFM.

$\S$ Università di Cagliari.

"CEMES/CNRS exhibit lower lasing threshold, ${ }^{18}$ and will result in improved power conversion efficiency in solar cells. ${ }^{19}$ Gold or magnetic tips on rods or on nanocrystals with other shapes ${ }^{14,15}$ can serve as preferential anchoring points for connecting nanocrystals to substrates, for their organization in solution into superstructures via linkage with molecules, or for their orientation under external biases.

The simplest heterostructure is the one based on the socalled core/shell system. In core/shell nanocrystals, an additional inorganic material is uniformly grown around a nanocrystal core, for disparate purposes. It can be used, for instance, to increase the robustness and the fluorescence efficiency of semiconductor nanocrystals, ${ }^{20-23}$ to create prototype quantum-confined structures as the quantum dot/ quantum well system, ${ }^{24}$ to tune the magnetic properties of the overall nanocrystal, ${ }^{25}$ or to provide a surface to which molecules can be attached easily. ${ }^{26}$ The design of more elaborate inorganic heterostructures, in which a second material is grown only on certain facets of a starting nanocrystal, requires a more accurate knowledge of the mechanism of growth under the various conditions. Cadmium chalcogenide nanocrystals can grow anisotropically in rods or in more complex shapes. ${ }^{6,10,11,27-29}$ When these materials form in the wurtzite structure in the presence of suitable surfactants, the lateral, nonpolar facets can have much lower growth rates than the basal, polar facets, and therefore the nanocrystals can develop preferentially along their unique $c$ axis. The higher reactivity of the tips of such anisotropic 
nanocrystals opens up the possibility to nucleate a second material exclusively at these locations. ${ }^{13,14}$

In the wurtzite structure, the absence of a plane of symmetry perpendicular to the $c$ axis implies that the 001 and the 001 facets are never equivalent. In $\mathrm{CdSe}$, the 001 facet exposes either $\mathrm{Cd}$ atoms with one dangling bond, or Se atoms with three dangling bonds, while the opposite is true for the $00 \overline{1}$ facet. We can therefore expect significant differences between the growth along the 001 and the $00 \overline{1}$ directions. This diversity was highlighted in the synthesis of arrow- and tadpole-shaped CdSe nanocrystals ${ }^{27,28}$ and in the lateral growth of sections of a semiconductor material on a starting semiconductor nanorod. ${ }^{13}$ Recent ab initio pseudopotential calculations on small CdSe wurtzite clusters suggest that the differences in reactivity among the various facets, even between the two polar facets perpendicular to the $c$ axis, can be related to differences in the passivating strength of surfactant molecules. ${ }^{30}$ In principle, it would be possible to exploit this concept to allow for the nucleation of a second material only on one polar facet of a wurtzite nanocrystal. In this communication, we report the selective growth of spherical PbSe nanocrystals on the tips of CdSe and CdS nanorods. The remarkable difference in reactivity among the various facets of the rods is clearly demonstrated for $\mathrm{CdS}$ as $\mathrm{PbSe}$ can be grown either on both tips of a rod or just on one tip, by carefully adjusting the synthesis conditions.

Nanorods of CdSe and CdS were prepared using octadecylphosphonic acid as promoter of anisotropic growth, according to the procedure of Peng and co-workers, although with minor changes (see Supporting Information). ${ }^{27,29}$ Sulfur or selenium were supplied to the reaction flask as trioctyl phosphine sulfide and trioctylphosphine selenide, respectively. After the synthesis, the rods were precipitated from the solution by the addition of methanol. This procedure yielded nanorods mixed with a gel due to unreacted $\mathrm{Cd}-$ phosphonate precursor. The separation of the nanorods from this gel is described in detail in the Supporting Information. The selective growth of PbSe nanocrystals on the nanorods was carried out by first dissolving the nanorods in diphenyl ether. To this solution a stock solution was injected which contained trioctylphosphine selenide, lead acetate, oleic acid, and diphenyl ether. These conditions were similar to those employed by Wehrenberg and co-workers to prepare monodisperse PbSe nanocrystals, ${ }^{31}$ although lower temperatures and lower concentrations of $\mathrm{PbSe}$ precursors were used here.

The selective growth of $\mathrm{PbSe}$ on both tips of $\mathrm{CdSe}$ nanorods (Figure 1a) was relatively straightforward. It was achieved by keeping the reaction temperature at $130^{\circ} \mathrm{C}$ and by injecting dropwise the stock solution of the $\mathrm{PbSe}$ precursors. This procedure yielded dumbbell-shaped, $\mathrm{PbSe}-$ $\mathrm{CdSe}-\mathrm{PbSe}$ hybrid nanocrystals (Figure $1 \mathrm{~b}$ ) and almost no isolated $\mathrm{PbSe}$ nanocrystals. Higher temperature decreased the selectivity in the end-growth since, under this circumstance, $\mathrm{PbSe}$ started forming also on the lateral facets of the rods.

The above conditions were extended to $\mathrm{CdS}$ nanorods (Figure 2a). In this case, only the homogeneous nucleation of PbSe nanocrystals in solution was observed, with no growth at all on the preformed semiconductor rods. Selective
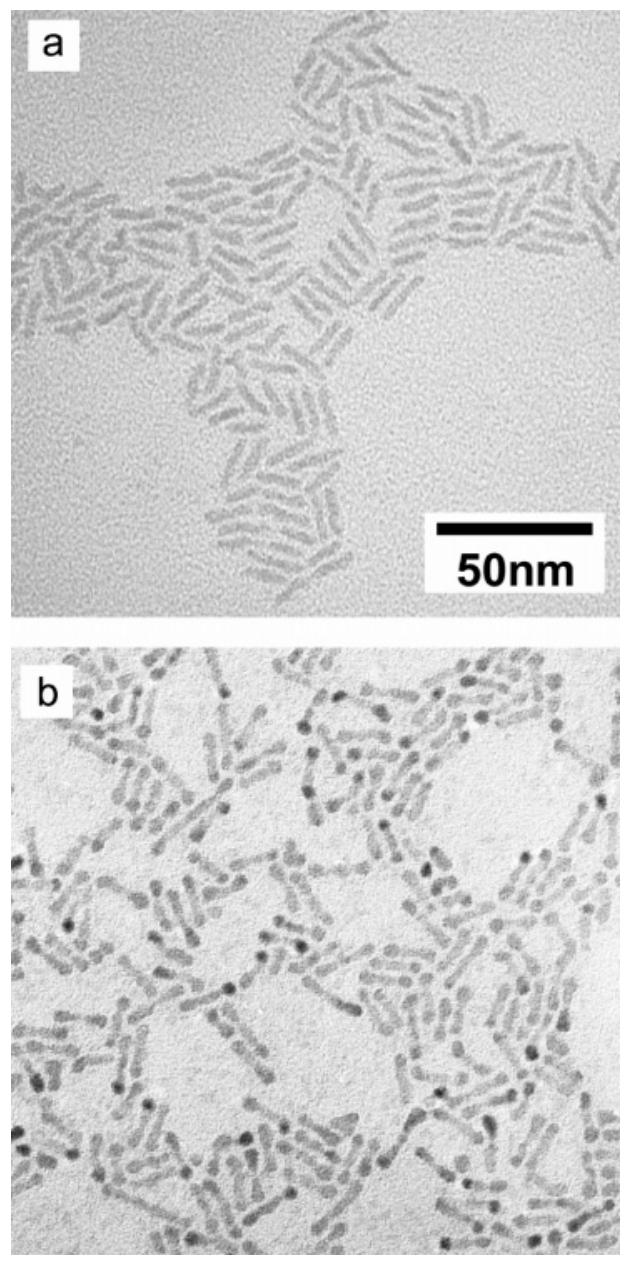

Figure 1. Low-resolution transmission electron microscopy (TEM) images of $\mathrm{CdSe}$ nanorods (a) and of dumbbell shaped $\mathrm{PbSe}-\mathrm{CdSe}-$ $\mathrm{PbSe}$ nanocrystal heterostructures (b).

growth of $\mathrm{PbSe}$ on $\mathrm{CdS}$ nanocrystals required both higher temperature and a fast injection of $\mathrm{PbSe}$ precursors (the injection temperature was $190{ }^{\circ} \mathrm{C}$, the growth temperature was $150{ }^{\circ} \mathrm{C}$ ), while growth on the lateral facets was never observed. These stronger conditions required to form $\mathrm{PbSe}-$ $\mathrm{CdS}-\mathrm{PbSe}$ dumbbells always led to the additional nucleation of isolated $\mathrm{PbSe}$ nanocrystals, from which dumbbells could be isolated via size-selective precipitation. In the final sample, the yield of dumbbells, estimated as the fraction of dumbbells with respect to other structures from statistical analysis of TEM images, was larger than 55\%. A lower concentration of $\mathrm{PbSe}$ precursors, delivered in the fast injection, reduced the nucleation of isolated nanocrystals drastically and promoted the preferential growth of PbSe on only one tip of the $\mathrm{CdS}$ rods (Figure 2b,c). In this case, at least $60-65 \%$ of the sample consisted of heterostructures showing a PbSe dot just at one tip of the $\mathrm{CdS}$ rod, and the remaining nanocrystals were mostly "asymmetric" dumbbells, in which one PbSe domain at one tip of the rods was usually much larger than the $\mathrm{PbSe}$ domain at the other tip.

Powder X-ray diffraction ${ }^{32}$ of spherical PbSe nanocrystals, $\mathrm{CdS}$ nanorods and $\mathrm{PbSe}-\mathrm{CdS}-\mathrm{PbSe}$ dumbbells are reported in Figure 3. The spectrum of the $\mathrm{CdS}$ nanorod sample indicates the presence of the hexagonal wurtzite structure. 

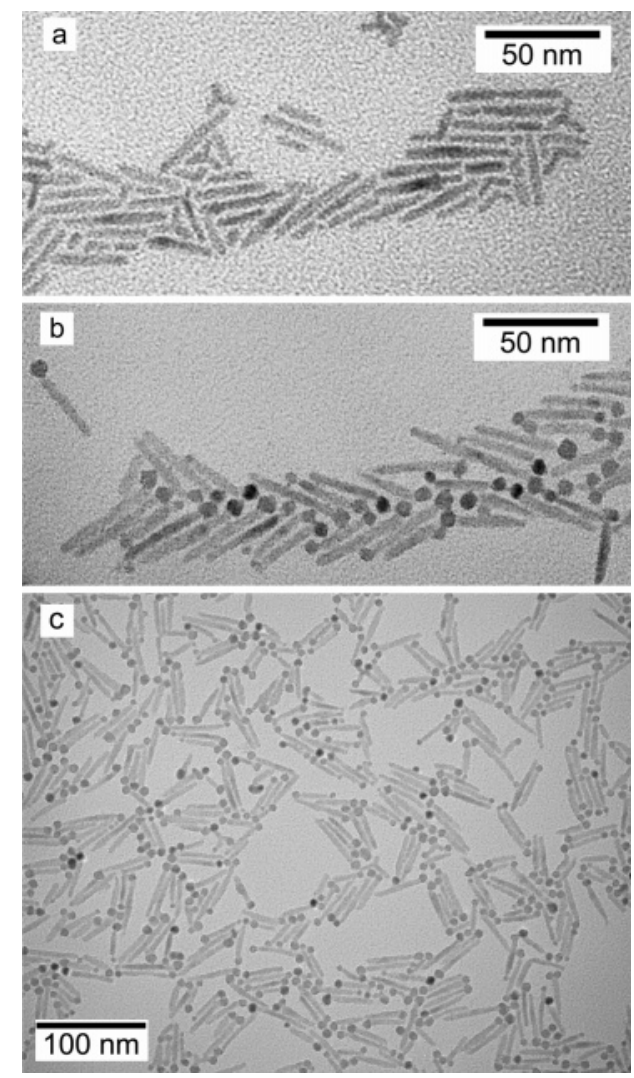

Figure 2. Low-resolution TEM images of CdS nanorods (a) and of $\mathrm{CdS}-\mathrm{PbSe}$ heterostructures with $\mathrm{PbSe}$ preferentially grown on one tip of the rods $(b, c)$.

The comparison with the diffraction pattern of bulk CdS shows a high degree of distortion of the intensity ratios due to shape anisotropy, ${ }^{6}$ which results in an enhancement of the reflections associated with crystalline domains along the $c$ axis. The spectrum of isolated dots indicates, on the other hand, that $\mathrm{PbSe}$ crystallizes in its typical rock-salt structure. The representative diffraction spectra for CdS-based dumbbells present only peaks which can be attributed either to the $\mathrm{PbSe}$ rock salt or to the $\mathrm{CdS}$ wurtzite structures. Therefore we conclude that $\mathrm{PbSe}$ grows on the nanorod tips in the rocksalt structure.

High-resolution $\mathrm{TEM}^{33}$ of CdSe- and CdS-based dumbbells (Figure 4) shows that in both cases PbSe crystallizes in the rock-salt structure on the tips of wurtzite nanorods, in agreement with the XRD results. The formation of nanocrystal heterostructures, with sections having remarkably different crystal structures, was shown recently for the case of gold tipped nanorods and tetrapods. ${ }^{14}$ Gold domains were reported to grow with no preferential crystallographic orientation with respect to the underlying wurtzite lattice. In our case, however, $\mathrm{PbSe}$ domains show a preferential orientation with respect to the substrate. In the majority of heterostructures based on $\mathrm{CdS}$ or on $\mathrm{CdSe}$, the 002 planes of PbSe rock salt were aligned with the 100 planes of $\mathrm{CdS}$ or CdSe wurtzite (Figure 4a,b).

The structure of the interface between $\mathrm{PbSe}$ and CdSe or between $\mathrm{PbSe}$ and $\mathrm{CdS}$ is not clear. Certainly, we cannot expect perfect epitaxial growth of a rock-salt island on top

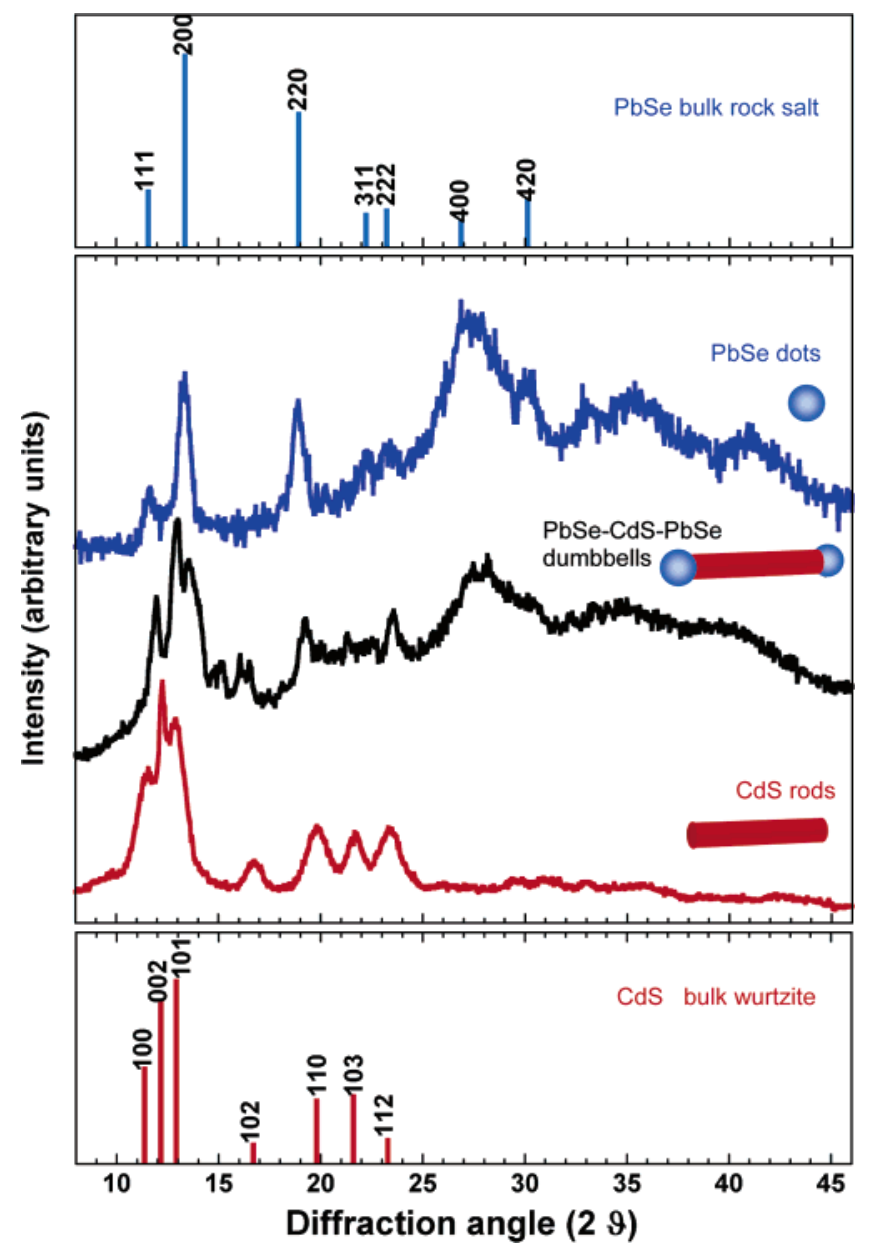

Figure 3. XRD spectra of $\mathrm{PbSe}$ dots, $\mathrm{CdS}$ rods, and $\mathrm{PbSe}-\mathrm{CdS}-$ $\mathrm{PbSe}$ dumbbells are compared with the diffraction pattern of bulk rock-salt $\mathrm{PbSe}$ and wurtzite $\mathrm{CdS}$.

of a wurtzite substrate, and therefore this interface should be highly defective, with considerable strain developing and with unsaturated dangling bonds left. The tips of the rods, prior to $\mathrm{PbSe}$ end-growth, do not show a sharp 001 or $00 \overline{1}$ termination (Figures 1a and 2a). Consequently, the wurtziterock-salt interface does not develop just along two facets, for instance between the $00 \overline{1}$ facet of wurtzite and the 100 facet of rock salt, but between several couples of facets between the two phases. This more complex interface might provide pathways for releasing and/or compensating the strain that can develop locally along a specific couple of facets.

The reasons underlying the large differences in reactivity among the various facets of the wurtzite structure, which allow for the selective growth on the tips of the rods, are still under investigation. So far, the indication is that this is driven by the difference in the binding energy of the surfactant molecules to the different facets. ${ }^{29,30}$ The results of our experiments do not allow us to recognize whether the 001 or the $00 \overline{1}$ facet is the most reactive. There is, however, evidence that the surfactants that are almost exclusively bound to the surface of nanocrystals are those that form strong complexes with $\mathrm{Cd}^{2+}$ ions in the growth solution but not with Se or $\mathrm{S}$ (for instance phosphonates ${ }^{29}$ or carboxylates ${ }^{12}$ ). Moreover, in semiconductors, as a 

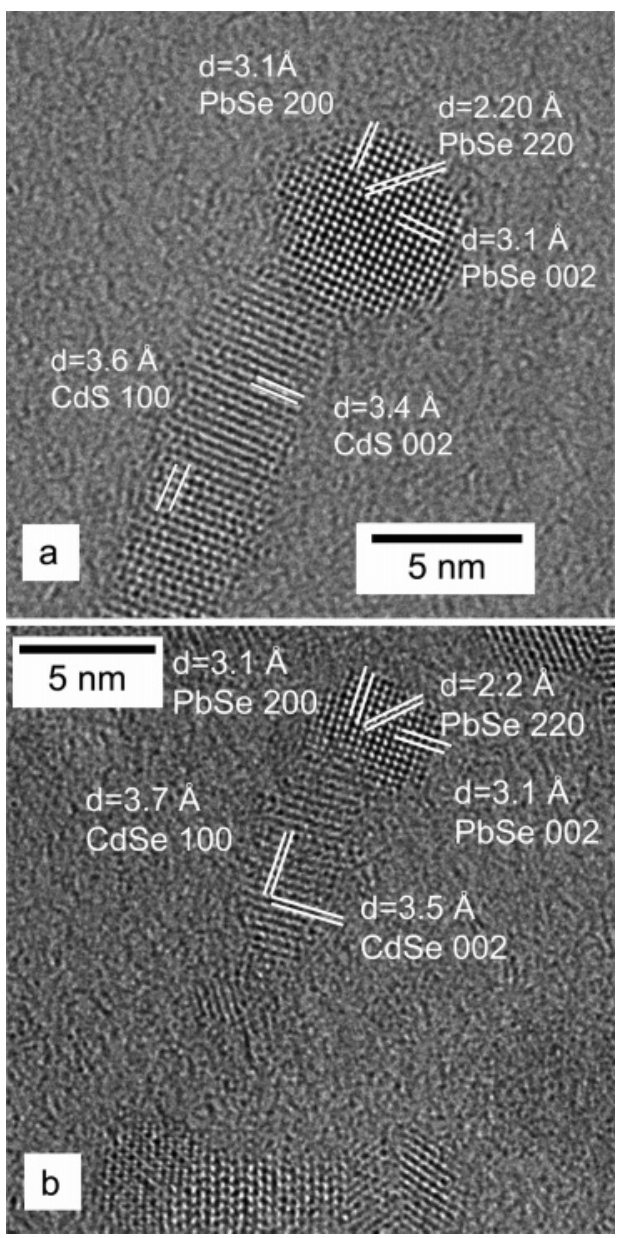

Figure 4. High-resolution TEM images of $\mathrm{PbSe}-\mathrm{CdS}-\mathrm{PbSe}$ (a) and $\mathrm{PbSe}-\mathrm{CdSe}-\mathrm{PbSe}$ dumbbells (b) with the corresponding interplanar distances. Image analysis shows that cubic PbSe dots grow at the tips of the CdS or CdSe hexagonal rods. HRTEM observations rule out heteroepitaxial growth of $\mathrm{PbSe}$ on the lateral facets of the rods.

consequence of surface reconstruction, ${ }^{34}$ the dangling bonds of surface cations $(\mathrm{Cd})$ are empty whereas the dangling bonds of surface anions $(\mathrm{S}, \mathrm{Se})$ are filled. If this concept is extended to the surface atoms of nanocrystals, one can argue that these Lewis base surfactants should bind exclusively to electron deficient surface $\mathrm{Cd}$ atoms and leave the surface $\mathrm{S}$ or $\mathrm{Se}$ atoms unpassivated. Therefore, we can speculate that incomplete or weak passivation of surface $\mathrm{Cd}$ atoms on a given facet is the main cause of instability for that facet, resulting in higher reactivity. Based on this assumption, the $00 \overline{1}$ facet is the most reactive one as it can expose $\mathrm{Cd}$ atoms with three dangling bonds each. On this facet, it is unlikely for all these dangling bonds to be passivated efficiently by bulky surfactant molecules, and therefore some of these bonds can serve as active sites for further growth.

Nanocrystal heterostructures represent unique systems in which the optical and electronic properties can be tuned by varying the chemical composition of their components and their mutual distance. Dumbbell-shaped nanocrystals, as the ones reported in this work, are composed of two PbSe quantum dots separated by a large potential barrier (either the $\mathrm{CdSe}$ or the $\mathrm{CdS}$ rod section). The height of this barrier

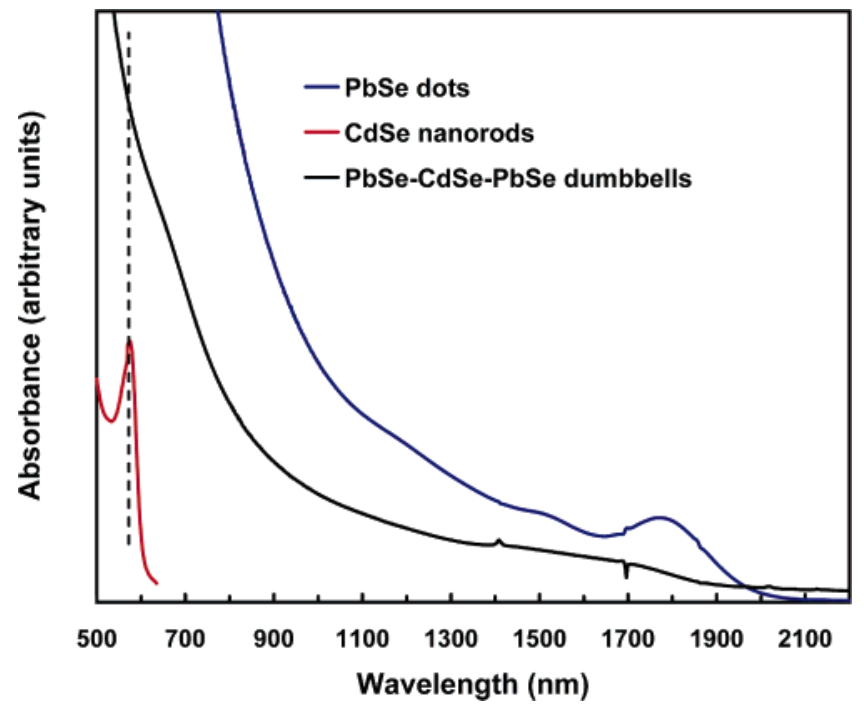

Figure 5. Optical absorption spectra of CdSe nanorods (red line), of spherical $\mathrm{PbSe}$ dots (blue line) and of $\mathrm{PbSe}-\mathrm{CdSe}-\mathrm{PbSe}$ dumbbells (black line), respectively.

can be adjusted by varying the diameter and the length of the rod. In Figure 5, the absorption spectra of a sample of CdSe nanorods, of a sample of spherical PbSe nanocrystals and of a corresponding $\mathrm{PbSe}-\mathrm{CdSe}-\mathrm{PbSe}$ dumbbell sample are shown, respectively. The exciton peak from the CdSe section is absent in the dumbbell spectrum, as the PbSe tips significantly modify the electronic structure of the starting rods. Although the carriers can be photogenerated over the whole dumbbell structure, we can expect that they are mainly localized at the PbSe tips. This peculiar property could be exploited, for instance, to align dumbbells under electric fields while they are photoexcited. The electric field would help to localize the photogenerated electrons and holes in the opposite PbSe tips, resulting in a large dipole moment that would help to align the rod under the field.

A heterostructure consisting of a nanorod with a single nanosphere attached to one tip (as the one reported in Figure 2) provides a particularly appealing structure. The potential exists for the tip of a magnetic material to act as a preferential anchoring point for the nanorod to a substrate, or as a "navigation" sensor.

This work shows that the development of approaches for the controlled growth of nanocrystalline heterostructures may significantly expand the range of applications of colloidal nanocrystals. The ease of fabrication of large amounts of nanocrystalline heterostructures by means of solution procedures fulfills an important requirement for their use as building blocks in nanotechnology.

Acknowledgment. This work was funded by the German research foundation (DFG, Emmy Noether program), the Deutscher Akademischer Austauschdienst (DAAD, Vigoni Funds), the Fonds der Chemischen Industrie, and by the Italian MIUR (FIRB and Vigoni Funds). We thank Dr. Mauro Lomascolo for helpful discussions.

Supporting Information Available: Description of the various nanocrystal syntheses, XRD characterization of the 
intermediate heterostructure with $\mathrm{PbSe}$ dots only at one tip of a $\mathrm{CdS}$ rod, low resolution TEM image of $\mathrm{PbSe}-\mathrm{CdS}-$ $\mathrm{PbSe}$ dumbbells, and optical spectra of $\mathrm{CdS}-\mathrm{PbSe}$ heterostructures. This material is available free of charge via the Internet at http://pubs.acs.org.

\section{References}

(1) Priester, C.; Lannoo, M. Phys. Rev. Lett. 1995, 75(1), 93-96.

(2) Morales, A. M.; Lieber, C. M. Science 1998, 279(5348), 208-211.

(3) Duan, X. F.; Lieber, C. M. Adv. Mater. 2000, 12(4), 298-302.

(4) Bjork, M. T.; Ohlsson, B. J.; Sass, T.; Persson, A. I.; Thelander, C.; Magnusson, M. H.; Deppert, K.; Wallenberg, L. R.; Samuelson, L. Nano Lett. 2002, 2(2), 87-89.

(5) Dick, K. A.; Deppert, K.; Larsson, M. W.; Martensson, T.; Seifert, W.; Wallenberg, L. R.; Samuelson, L. Nature Mater. 2004, 3(6), 380-384

(6) Peng, X. G.; Manna, L.; Yang, W. D.; Wickham, J.; Scher, E.; Kadavanich, A.; Alivisatos, A. P. Nature 2000, 404(6773), 59-61

(7) Kim, Y. H.; Jun, Y. W.; Jun, B. H.; Lee, S. M.; Cheon, J. W. J. Am. Chem. Soc. 2002, 124(46), 13656-13657.

(8) Lee, S. M.; Jun, Y. W.; Cho, S. N.; Cheon, J. J. Am. Chem. Soc. 2002, 124(38), 11244-11245.

(9) Puntes, V. F.; Zanchet, D.; Erdonmez, C. K.; Alivisatos, A. P. J. Am. Chem. Soc. 2002, 124(43), 12874-12880.

(10) Jun, Y. W.; Lee, S. M.; Kang, N. J.; Cheon, J. J. Am. Chem. Soc. 2001, 123(21), 5150-5151.

(11) Manna, L.; Milliron, D. J.; Meisel, A.; Scher, E. C.; Alivisatos, A. P. Nature Mater. 2003, 2(6), 382-385.

(12) Yu, W. W.; Wang, Y. A.; Peng, X. G. Chem. Mater. 2003, 15(22), $4300-4308$.

(13) Milliron, D. J.; Hughes, S. M.; Cui, Y.; Manna, L.; Li, J. B.; Wang, L. W.; Alivisatos, A. P. Nature 2004, 430(6996), 190-195.

(14) Mokari, T.; Rothenberg, E.; Popov, I.; Costi, R.; Banin, U. Science 2004, 304(5678), 1787-1790.

(15) Gu, H. W.; Zheng, R. K.; Zhang, X. X.; Xu, B. J. Am. Chem. Soc. 2004, 126(18), 5664-5665.

(16) Hu, J. T.; Li, L. S.; Yang, W. D.; Manna, L.; Wang, L. W.; Alivisatos, A. P. Science 2001, 292(5524), 2060-2063.

(17) Rothenberg, E.; Ebenstein, Y.; Kazes, M.; Banin, U. J. Phys. Chem. B 2004, 108(9), 2797-2800.
(18) Kazes, M.; Lewis, D. Y.; Ebenstein, Y.; Mokari, T.; Banin, U. Adv. Mater. 2002, 14(4), 317-+.

(19) Huynh, W. U.; Dittmer, J. J.; Alivisatos, A. P. Science 2002, 295(5564), 2425-2427.

(20) Hines, M. A.; Guyot-Sionnest, P. J. Phys. Chem. 1996, 100(2), 468471.

(21) Dabbousi, B. O.; Rodriguez-Viejo, J.; Mikulec, F. V.; Heine, J. R.; Mattoussi, H.; Ober, R.; Jensen, K. F.; Bawendi, M. G. J. Phys. Chem. B 1997, 101(46), 9463-9475.

(22) Peng, X. G.; Schlamp, M. C.; Kadavanich, A. V.; Alivisatos, A. P. J. Am. Chem. Soc. 1997, 119(30), 7019-7029.

(23) Cao, Y. W.; Banin, U. J. Am. Chem. Soc. 2000, 122(40), 96929702.

(24) Eychmuller, A.; Mews, A.; Weller, H. Chem. Phys. Lett. 1993, 208(1-2), 59-62.

(25) Zeng, H.; Li, J.; Wang, Z. L.; Liu, J. P.; Sun, S. H. Nano Lett. 2004, 4(1), 187-190.

(26) Cao, Y. W.; Jin, R.; Mirkin, C. A. J. Am. Chem. Soc. 2001, 123(32), 7961-7962.

(27) Peng, Z. A.; Peng, X. G. J. Am. Chem. Soc. 2001, 123(7), 13891395.

(28) Manna, L.; Scher, E. C.; Alivisatos, A. P. J. Am. Chem. Soc. 2000, 122(51), 12700-12706.

(29) Peng, Z. A.; Peng, X. G. J. Am. Chem. Soc. 2002, 124(13), 33433353.

(30) Puzder, A.; Williamson, A. J.; Zaitseva, N.; Galli, G.; Manna, L.; Alivisatos, A. P. Nano Lett. 2004, 4(12), 2361-2365.

(31) Wehrenberg, B. L.; Wang, C. J.; Guyot-Sionnest, P. J. Phys. Chem. $B$ 2002, 106(41), 10634-10640.

(32) XRD spectra were collected using a D500 Siemens diffractometer equipped with Mo Ka radiation and a graphite monochromator on the diffracted beam. Phase identification was performed according to the Powder Diffraction File database [PDF-2 File, JCPDS International Center for Diffraction Data, 1601 Park Lane, Swarthmore, PA 1998].

(33) HRTEM measurements were performed at the CEMES/CNRS Laboratory in Toulouse on a FEI SACTEM microscope, equipped with a field emission electron source operating at $200 \mathrm{kV}$ and with a spherical aberration corrector.

(34) Duke, C. B. Chem. Rev. 1996, 96, 1237-1259.

\section{NL048060G}

